\title{
Effectiveness Evaluation of Additional Risk Minimization Measures for Adolescent Use of Aripiprazole in the European Union: Results from a Post-Authorization Safety Study
}

\author{
Wally Landsberg ${ }^{1}$ - Imad Al-Dakkak ${ }^{2}$ - Antonia Coppin-Renz ${ }^{3}$ - Uli Geis ${ }^{3}$. \\ Timothy Peters-Strickland ${ }^{4} \cdot$ Emiel van Heumen $^{3} \cdot$ Mirza Rahman $^{4}$
}

Published online: 18 April 2018

(C) The Author(s) 2018

\begin{abstract}
Introduction Two risk minimization (RM) tools-a healthcare professional frequently asked questions (HCP-FAQs) brochure and a patient/caregiver information brochure (PCIB) - were developed for HCPs and for adolescents (aged $\geq 13$ years) receiving aripiprazole for bipolar I mania and their caregivers.

Objectives This study evaluated the effectiveness of these RM tools in improving the awareness and education of HCPs and patients/caregivers.

Method The RM tools were distributed to HCPs (identified in agreement with the marketing authorization holder [MAH] and local regulatory authorities), who in turn distributed the PCIBs to patients/caregivers. A web-based survey was then conducted targeting HCPs and patients/caregivers.

Results The response rate was low: 118 of 23,282 invited HCPs and 16 patients/caregivers completed the survey. Overall, 42\% (49/118) of HCP respondents were aware of aripiprazole RM tools; of these, 59\% (29/49) of HCPs read them at least once and 66\% (19/29) of these used the RM tools while
\end{abstract}

Electronic supplementary material The online version of this article (https://doi.org/10.1007/s40264-018-0662-2) contains supplementary material, which is available to authorized users.

Wally Landsberg

wlandsberg@otsuka-europe.com

1 Otsuka Europe Development and Commercialization Ltd., Gallions, Wexham Springs, Framewood Road, Wexham SL3 6PJ, UK

2 Pope Woodhead \& Associates Ltd., Cambridgeshire PE27 5BZ, UK

3 Otsuka Europe Development and Commercialization Ltd., Europa-Allee 52, 60327 Frankfurt am Main, Germany

4 Otsuka Pharmaceutical Development and Commercialization Inc., Princeton, NJ 08540, USA discussing the benefit-risk profile of aripiprazole with patients/caregivers. In total, 30 of the 118 HCPs (25\%) were aware of the PCIB, and 26 distributed it to their patients/caregivers, whereas seven HCPs advised them to read the brochure. Overall, 15 of the 16 patients/caregivers were aware of the $\mathrm{PCIB}$, and $13 \mathrm{read} / \mathrm{referred}$ to it. Of these, 12 found the PCIB useful, and five monitored their weight while receiving aripiprazole and reported potential risks immediately to their HCP. Conclusion The response rate to the survey was low, and the tools displayed limited utility and effectiveness in improving awareness and education in a small number of responders. Therefore, the aripiprazole risk management plan was amended, and the tools were discontinued.

\section{Key Points}

This web-based survey study evaluated the effectiveness of additional risk minimization measures (aRMMs) and the impact of these aRMMs on the awareness and knowledge levels of recipients-healthcare professionals (HCPs) and patients and their caregivers.

The risk minimization tools had limited reach and/or response and thus their utility and effectiveness in educating the target audience was also limited. However, within this limitation, the tools were found to be useful among patients and caregivers who received them from their treating HCPs.

The survey results provided insights and recommendations that can be incorporated into similar studies in the future to enable a wider and more targeted reach to prescribers and users while improving clinical safety outcomes. 


\section{Introduction}

Bipolar I (BPI) disorder is a common neuropsychiatric condition characterized by recurring episodes of mania and depression, or mixed episodes [1]. The global prevalence of all types of bipolar disorders is approximately 4\% [1], and the estimated prevalence of BPI ranges from 0.2 to $1.5 \%$ [2]. Most patients with BPI report an onset of mood symptoms during adolescence or early adulthood, i.e., between 15 and 25 years of age [3].

Aripiprazole (ABILIFY ${ }^{\circledR}$ [Otsuka Pharmaceutical Europe Ltd., UK]) is an atypical antipsychotic licensed in the EU for the treatment (for a period of up to 12 weeks) of moderate-to-severe manic episodes in adolescents (aged $\geq 13$ years) with BPI [4]. Aripiprazole has proven efficacy in BPI mania, but-as with other drugs-its use is associated with adverse drug reactions (ADRs), of which extrapyramidal symptoms (EPS), fatigue, weight gain, and somnolence require additional attention in the adolescent age group [4-6]. Besides the established ADRs, other unknown ADRs of concern could occur at a low frequency. Such ADRs might be discovered following long-term use in a larger patient population in a real-world setting. Therefore, marketing authorization holders (MAHs) in the $\mathrm{EU}$ are required to submit a risk management plan (RMP) when seeking an application for the approval of a new drug product. This ensures the benefits of a drug outweigh its risks throughout its use by the intended population. The MAHs are also required to have risk minimization measures (RMMs) in place, such as the summary of product characteristics and patient information leaflet, and update these periodically, as per regulatory requirements [7].

Typically, most safety concerns are addressed by routine RMMs. However, these may not be sufficient for certain drugs; specific safety issues - such as the potential for medication errors, off-label use, and safety concerns in specific populations (e.g. pediatric and adolescent patients) - may need to be addressed [7, 8]. In such cases, the MAH is required to develop additional RMMs (aRMMs) that might include controlled access programs and/or educational material for healthcare professionals (HCPs) and patients, such as patient alert cards, frequently asked questions (FAQs), and patient/caregiver information brochures (PCIBs) [7, 8].

As part of the marketing authorization approval of aripiprazole for the treatment of moderate-to-severe manic episodes in adolescents with BPI, the MAH developed an RMP supported by aRMMs. Recent EU pharmacovigilance legislation has made it mandatory for MAHs and respective local regulatory authorities to monitor the effectiveness of aRMMs periodically to ensure the objectives of the aRMMs are met and gaps/opportunities for better implementation are identified [9].
This post-authorization safety study (PASS) evaluated the effectiveness of the aripiprazole aRMMs, which included two risk minimization (RM) tools: the HCP-FAQs brochure and the PCIB. The key objective of this study was to establish a baseline level of tool usage and knowledge and the resulting relevant behaviors of recipients. The HCP-FAQs brochure was designed to inform HCPs about the indicated age range, dose, and duration of aripiprazole treatment before they prescribe it to adolescents with BPI, highlight ADRs of concern, and to remind them to apprise patients/caregivers of potential ADRs and distribute the PCIB. The PCIB was designed to educate patients/caregivers about aripiprazole administration and remind them to be vigilant for ADRs and to spontaneously report ADRs to their treating HCPs. The broad content of these tools was approved by the European Medicines Agency Pharmacovigilance Risk Assessment Committee (EMA-PRAC).

The RM tools were initially distributed in 2013 to the locally agreed lists of HCPs from EU member states, where aripiprazole is approved for the treatment of adolescents with BPI mania and was subsequently available for prescription in this indication. The RM tools were made available to the HCPs upon request from the MAH's medical information function. The final translated content, method of distribution, and HCP lists for distribution of $\mathrm{RM}$ tools were agreed upon at the country level between local representatives of the MAH and applicable local regulatory authorities.

Several studies (mandated or voluntary) have evaluated safety outcomes and effectiveness of drugs, and an abundance of knowledge and experience is available regarding the methodology and guidance for such studies [10]. However, studies evaluating the effectiveness of aRMMs are relatively new, and knowledge on the approaches used to conduct these studies and their success determinants is limited [11], as is the number of published studies [12, 13].

One of the approaches to assess the effectiveness of aRMMs as per the Good Pharmacovigilance Practice (GVP) Module XVI framework includes evaluation of process indicators such as measurement of tool distribution, utility, acquired knowledge, as well as resulting selfreported behavior, and outcome indicators such as a reduction in the occurrence and severity of ADRs [7]. Generally, survey studies are considered one of the conventional methods to measure process indicators. However, they have some disadvantages, including generalizability (sample may not be representative of the entire target population) and bias regarding selection of the study population [11].

To date, examples of surveys conducted in the EU to evaluate the effectiveness of aRMMs process indicators are limited. In addition, survey-based studies evaluating the effectiveness of aRMMs with an aim to assess knowledge 
of risks and off-label use are classified as PASS per GVP Module VIII. Thus, such survey studies, if conducted in the $\mathrm{EU}$, are required to follow stringent regulatory guidelines [11].

To the best of our knowledge, this is one of the first survey studies to be conducted in the EU for BPI disorder in adolescents that evaluates the effectiveness of aRMMs. We believe this study provides a unique perspective from the viewpoint of HCPs and patients/caregivers regarding the effectiveness of aRMMs, the operational challenges faced by MAHs in implementing survey studies in the EU, and possible corrective measures if the aRMMs are deemed ineffective.

\section{Methods}

\subsection{Study Design}

This was a non-interventional, cross-sectional, surveybased study. It comprised three sets of web-based surveys implemented using a proprietary tool called SafetyGauge (developed by Pope Woodhead \& Associates Ltd., UK) to assess the effectiveness of aRMMs implemented to improve the awareness and knowledge among HCPs and patients/caregivers on the appropriate use of aripiprazole for the treatment of BPI mania in adolescents. The webbased surveys included surveys for HCPs, patients, and caregivers. The caregiver survey was very similar to that for patients but was designed to enable caregivers to provide information on behalf of patients. Ethics committee approval was obtained in Slovenia (Komisija Republike Slovenije za Medicinsko Etiko), Spain (Comité Ético de Investigación Clínica del Area de Salud de Burgos y Soria), Germany (Ethik-Kommission bei der Landesärztekammer Hessen), and Italy (Comitato Etico Azienda Ospedaliero Universitaria di Cagliari) as per local requirements. For the remaining countries, approval from an ethics committee was not required to conduct the surveys.

\subsection{Study Population}

The study population included adolescents (aged $\geq 13$ years) who had been or were being treated with aripiprazole for BPI mania, their caregivers, and treating HCPs. In total, 23,282 invitations to participate in the survey were initially issued to HCPs. Patients/caregivers were directly recruited via their HCPs only. An honorarium (at fair market value) was offered to HCPs for their time and paid accordingly after survey completion. Patients and caregivers were not offered compensation.

\subsection{Recruitment}

The survey was conducted in 12 countries: the UK, Germany, Spain, Italy, Sweden, Norway, Ireland, Austria, Portugal, Greece, Slovenia, and Denmark. However, no HCPs from Slovenia or Denmark responded to the survey.

Data collection was performed in two stages: between 27 July 2014 and 9 July 2015 and between 10 July 2015 and 4 January 2016.

Lists of HCPs who would have been sent the aRMMs were obtained from the IMS Healthcare Organization Services Database and/or local country affiliates. During the first data collection period, HCP distribution lists intended for invitation were randomized, per protocol [14], in blocks of 200. The randomization process used at the beginning of the study involved selecting at random 200 HCPs from the full list per country, then inviting those HCPs to participate. Initially, invitations in the local language were mailed to HCPs; if no response was received, additional mailings were sent, with up to a total of three invitations to participate in the web-based survey. Only email addresses of HCPs from Spain were available.

A second round of randomization, without replacement, was conducted. Since this approach resulted in a very low response rate, the randomization approach was abandoned, and the invitation was posted to the full HCP list en masse in each country. However, the recruitment improved only marginally despite obtaining more complete data on HCPs (including email addresses, where possible), introducing three rounds of postal invitations, and the replacement of paper-based HCP contracts with a "click to consent" button at the start of the web-based survey.

The HCPs, patients, and caregivers who consented were provided with unique log-on credentials and a link to the local language secure website, pertinent to the survey they were asked to complete.

\subsection{Study Endpoints}

The primary objectives of the study were to (1) determine the proportion of HCPs who were aware of the existence of the RM tools and how they were accessed; (2) determine when, how, and by whom these tools were used; (3) determine the knowledge level and comprehension of the identified key risks associated with aripiprazole when used for the treatment of BPI mania in adolescents (i.e., EPS, weight gain, somnolence, and fatigue); and (4) evaluate HCP, patient, and caregiver behavior using targeted questions and hypothetical risk scenarios. The study also evaluated the effect of the RM tools on minimizing realworld risks such as an inappropriate starting dose of aripiprazole and patient age (i.e., in patients aged $<13$ years). 
The variables used for primary endpoint analysis are presented in Table 1 in the Electronic Supplementary Material (ESM).

\subsection{Data Analysis}

The survey primarily comprised multiple-choice questions (see the ESM), with conditional branching depending on the answers provided in the case of questions assessing knowledge, and optional descriptive comments in a small number of sections. The sample size was initially driven by the estimated size of the adolescent patient population potentially receiving aripiprazole for the treatment of BPI mania, with an acceptable margin of error $(<20 \%)$ in the population of interest. The full dataset included all participants who completed the survey using the SafetyGauge Platform, which was developed for building interactive surveys and deploying logical branching based on participant replies. Descriptive statistics were used to summarize responses to the survey questions.

\section{Results}

\subsection{Healthcare Professional Survey}

A total of 23,282 initial invitations to participate in the survey were issued to HCPs. The response rate to the survey during the first data collection period was considerably lower (1.4\%) than that assumed in the study protocol $(30 \%)$. After timelines were extended and revised recruitment activities implemented, the response rate in the second data collection period improved slightly to $2.3 \%$.

Table 1 Recruitment of healthcare professionals across the EU

\begin{tabular}{lrl}
\hline Country & Target no. of HCPs & Total HCPs recruited $(N)$ \\
\hline Spain & 1508 & 42 \\
UK & 2188 & 18 \\
Italy & 10,600 & 15 \\
Greece & 195 & 12 \\
Germany & 1930 & 8 \\
Portugal & 1445 & 8 \\
Norway & 478 & 6 \\
Sweden & 761 & 4 \\
Ireland & 34 & 3 \\
Austria & 1661 & 2 \\
Slovenia $^{\mathrm{a}}$ & 35 & 0 \\
Denmark $^{\mathrm{a}}$ & 780 & 0 \\
\hline
\end{tabular}

${ }^{a}$ None of the HCPs invited participated in the survey

$H C P$ healthcare professional, EU European Union
In total, $118 \mathrm{HCPs}$ responded to the survey. The HCPs most commonly treated patients with BPI at community teaching hospitals (32/118 [27\%]) and university teaching hospitals (25/118 [21\%]). Most of the HCPs were from Spain (42/118 [36\%]) and were general psychiatrists (74/ 118 [63\%]) and child/adolescent psychiatrists (40/118 [34\%]). Table 1 shows the overall recruitment of HCPs across ten countries in the EU.

More than half of the HCPs $(77 / 117$ [66\%]) treated $>$ 20 patients with aripiprazole for all indications (Fig. 1a). However, for the BPI mania indication, most of the HCPs treated only one to five patients with aripiprazole (Fig. 1b).

Among the HCPs with substantial experience (i.e. treated $>20$ patients) in prescribing aripiprazole, about half were aware of the HCP-FAQs brochure (14/25) and PCIB (13/25) (Fig. 2). Overall, 42\% (49/118) and 41\% (48/117) of the responding HCPs were aware of the existence of the HCP-FAQs brochure and PCIB, respectively (Fig. 2). Of

(a)
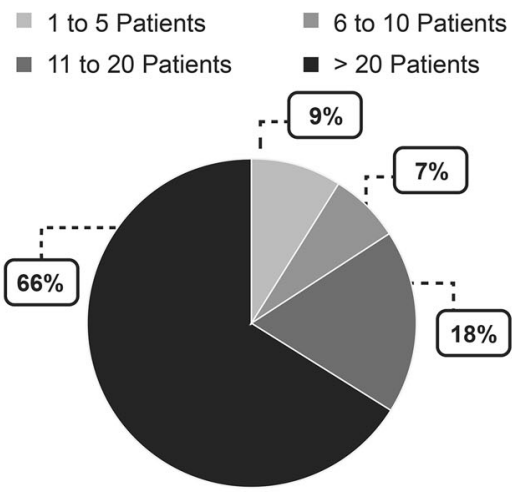

Total HCPs $=117^{*}$

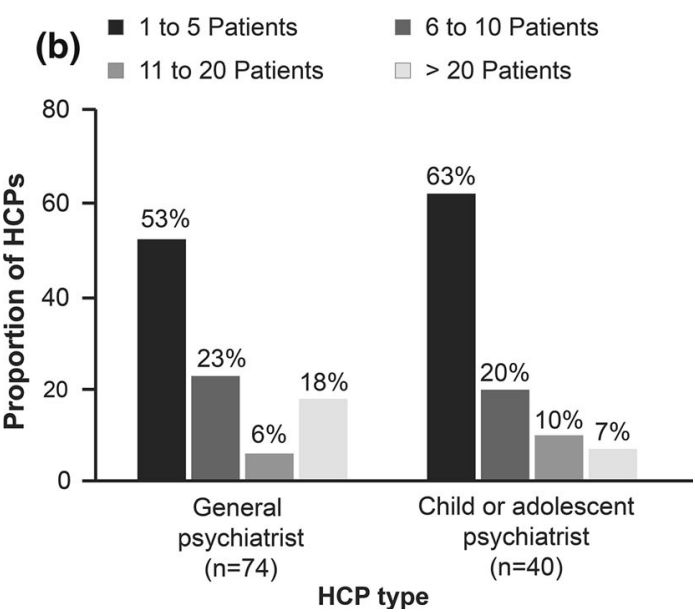

Fig. 1 (a) Proportion of HCPs based on experience of number of patients treated with aripiprazole; (b) HCP type stratified by number of patients they treated with aripiprazole (other HCPs are not shown as only four were recruited, three of which had experience treating one to five patients and only one had experience treating 11-20 patients with aripiprazole). *Data for one HCP are missing 


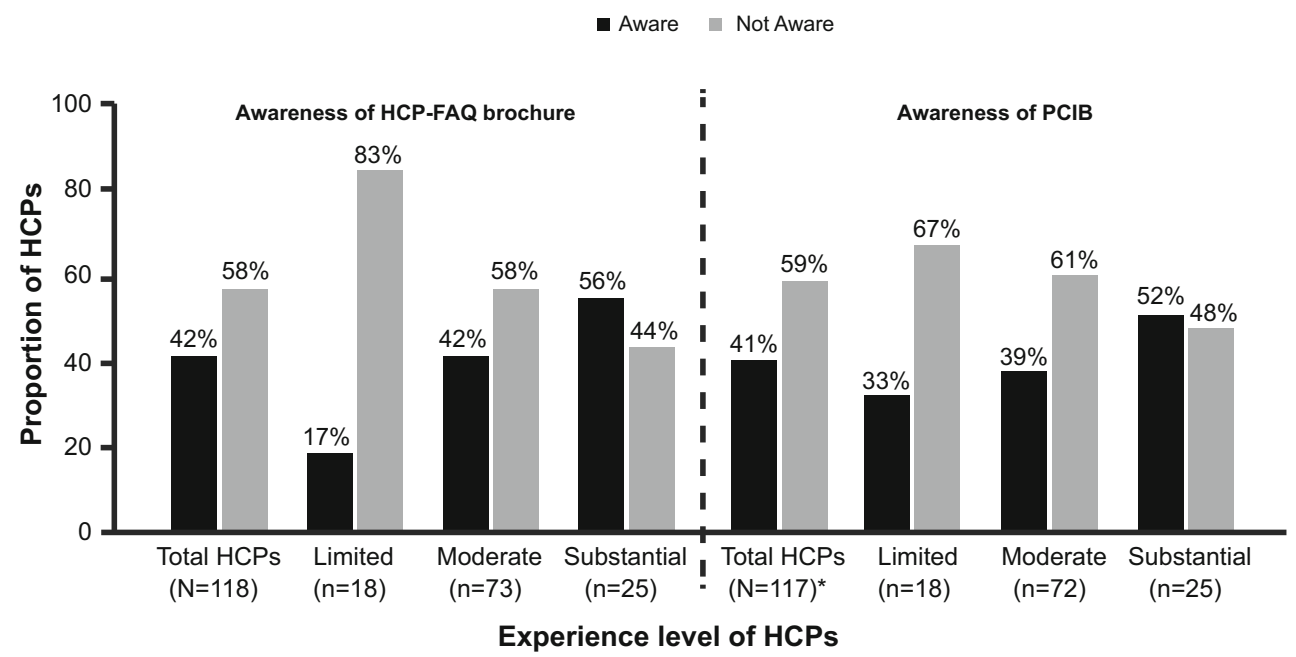

Fig. 2 Awareness of risk minimization tools among healthcare professionals (HCPs). *Data for one HCP were missing. The sum of HCP categories for HCP-FAQ brochure and PCIB had ' 1 missing value' and ' 1 error value'. HCPs were categorized as having "limited" experience if they treated up to ten patients with aripiprazole and up to five adolescents diagnosed with bipolar I (BPI) disorder, "moderate" experience if they had treated more than
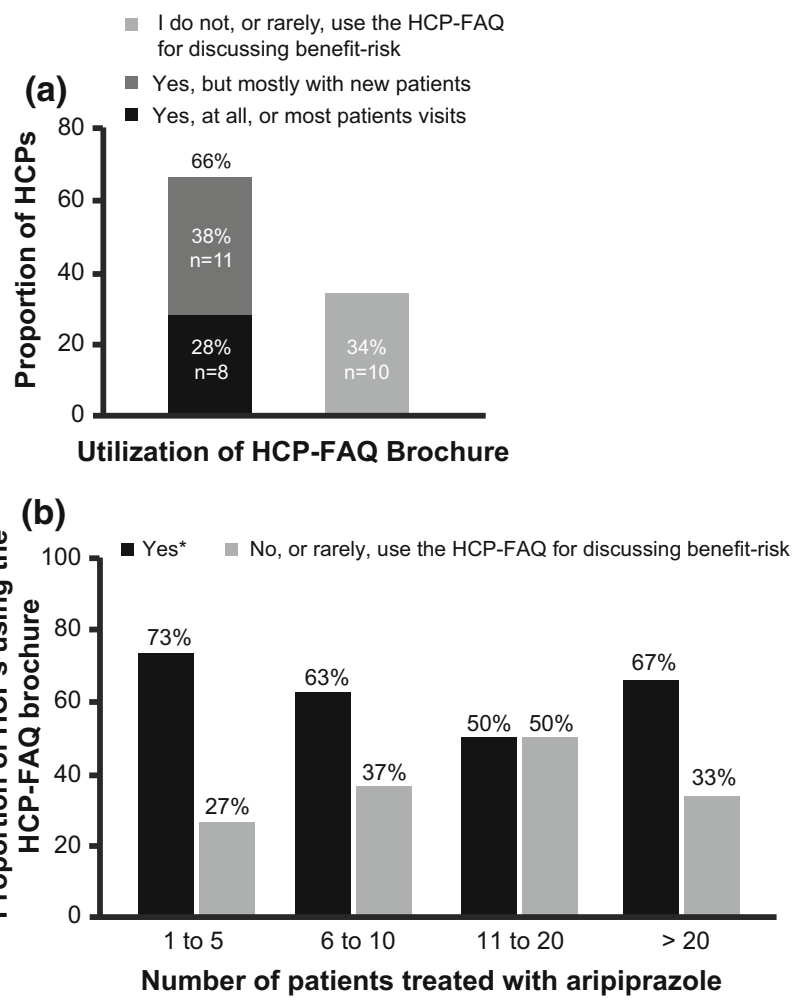

Fig. 3 Utilization of risk minimization tools among healthcare professionals (HCPs). (a) Proportion of HCPs who used the HCP-FAQ brochure when discussing the benefit-risk profile of aripiprazole with patients. (b) Proportion of HCPs (by experience) who used HCP-FAQ brochure when discussing benefit-risk profile of aripiprazole with patients/caregivers. *Data were combined for responses "yes, at all, or most, patient visits" and "yes, but mostly with new patients only". $F A Q$ frequently asked questions, $P C I B$ patient/caregiver information brochure ten patients with aripiprazole and up to ten adolescents diagnosed with BPI disorder, and "substantial" experience if they had treated more than ten patients with aripiprazole and at least 20 adolescents diagnosed with BPI. BPI bipolar I, $F A Q$ frequently asked questions, $H C P$ healthcare professional, $P C I B$ patient/caregiver information brochure

the $49 \mathrm{HCP}$ respondents, only 59\% $(n=29)$ reported receiving a copy of the HCP-FAQs brochure and reading it fully, or partially, at least once. Of these 29 HCPs, only $24 \%(n=7)$ of HCPs were aware of the correct aripiprazole dosage regimen for treating adolescents with BPI mania, and 66\% ( $n=19)$ were using the HCP-FAQs brochure when discussing the benefit-risk profile of aripiprazole with patients/caregivers (Fig. 3a).

The HCPs who treated the least number of patients (one to five patients) used the HCP-FAQs brochure more frequently while discussing the benefit-risk profile of aripiprazole with patients/caregivers (Fig. 3b). Of the 19 HCPs who used the HCP-FAQs brochure while discussing the benefit-risk profile of aripiprazole with patients/caregivers, $18(95 \%)$ used at least one of the RM tools while reviewing safety information with patients/caregivers.

Of the 89 HCPs who did not read the HCP-FAQs brochure, $30 \%(n=27)$ had knowledge of the correct dosage regimen of aripiprazole for the treatment of adolescents with moderate-to-severe BPI mania.

\subsection{Patients/Caregivers Survey}

Of 30 HCPs who reported receiving copies of the PCIB, 26 (87\%) distributed the PCIB to patients/caregivers, and only seven $(23 \%)$ advised patients/caregivers to read the PCIB.

In total, 16 patients/caregivers responded to the survey. None of the responding patients were aged $<13$ years when they first received aripiprazole treatment, and all were apparently treated within the authorized indication 
Fig. 4 Awareness and utilization of the patient/caregiver information brochure (PCIB) among patients/caregivers. Both patients and caregivers received the PCIB most commonly in printed format
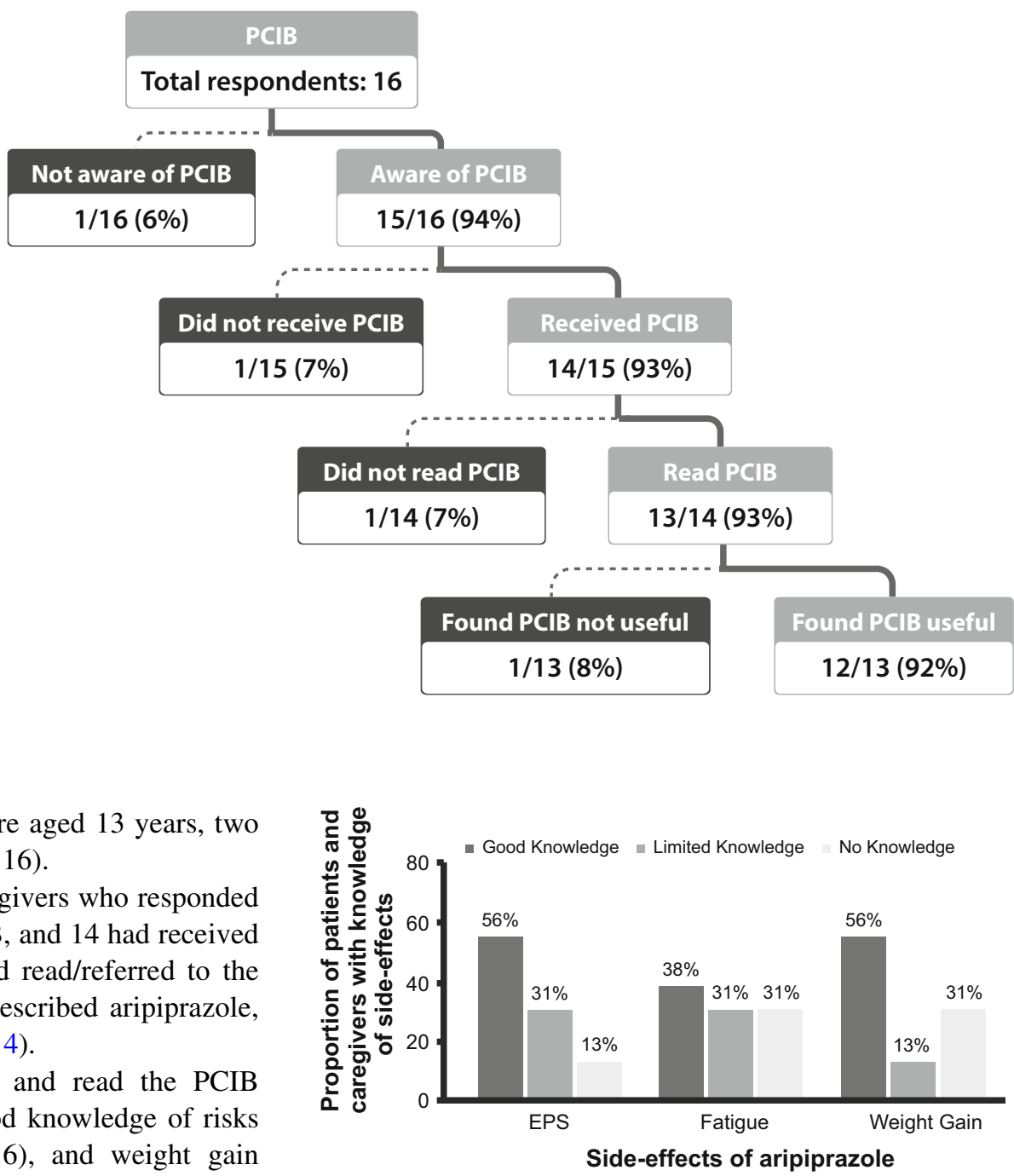

Fig. 5 Knowledge level of patients/caregivers regarding side effects associated with aripiprazole treatment $(N=16)$. Knowledge level of patients/caregivers was assessed based on the responses to the questions regarding extrapyramidal symptoms (EPS) ("I may get twitching/jerking [uncontrollable]" and "has read about EPS"), fatigue ("has read about fatigue" and "I may feel more tired than usual"), and weight gain ("told or read that weight may increase" and "told that weight may increase"). Patients/caregivers with two "yes" responses to the respective questions related to each side effect are considered to have "good knowledge." Patients with one "yes" response have "limited knowledge," and those with no "yes" responses have "no knowledge." $A E$ adverse event

\section{Discussion}

This is one of the first effectiveness evaluation studies of RM tools in patients with BPI mania within the EU. Before starting the study, the MAH directly distributed the HCPFAQs brochure and PCIB to individual HCPs, as per distribution lists agreed with local regulatory authorities. The aim was to inform HCPs and educate them about the need 
Table 2 Behavior of patients/caregivers around side effects associated with aripiprazole treatment

\begin{tabular}{|c|c|c|c|c|}
\hline Answers selected by patient/caregivers, $N=16$ & Weight gain & EPS & Fatigue & Somnolence \\
\hline Tell my doctor at the next visit & $8(50)$ & $3(19)$ & $8(50)$ & $4(25)$ \\
\hline $\begin{array}{l}\text { Tell my doctor at the next visit and tell my doctor immediately } \\
\text { and wait and see if it changes (selecting two options) }\end{array}$ & $1(6)$ & $1(6)$ & $1(6)$ & $1(6)$ \\
\hline Tell my doctor at the next visit and tell my doctor immediately & NS & $1(6)$ & $2(13)$ & $2(13)$ \\
\hline Tell my doctor immediately & $1(6)$ & $8(50)$ & $2(13)$ & $5(31)$ \\
\hline Tell my doctor at the next visit and let the patient continue taking aripiprazole & $1(6)$ & NS & $1(6)$ & NS \\
\hline $\begin{array}{l}\text { Tell my doctor at the next visit and tell my doctor immediately } \\
\text { and let the patient continue taking aripiprazole (selecting two options) }\end{array}$ & $1(6)$ & NS & NS & $1(6)$ \\
\hline Stop taking aripiprazole treatment & $1(6)$ & $1(6)$ & NS & NS \\
\hline Wait and see if it changes & $1(6)$ & $1(6)$ & NS & $1(6)$ \\
\hline I don't know & $2(13)$ & $1(6)$ & NS & $2(13)$ \\
\hline
\end{tabular}

Data are presented as $n(\%)$

EPS extrapyramidal symptoms, $N S$ option not selected

to take certain actions or to adopt practices related to aripiprazole treatment in adolescents with moderate-tosevere BPI mania. Nonetheless, the challenges faced by the MAHs seemed to affect the distribution of the RM tools to the intended audience and their subsequent participation in the survey, which compromised the quality of this study evaluating the effectiveness of RM tools.

The study results showed that most of the surveyed HCPs were unaware of the existence of the RM tools, but most of the HCPs who had received and read the RM tools considered them to be either very or quite useful. The patient/caregiver survey responses indicated the PCIBs had a good impact in terms of education and awareness; however, given the small number of participants, these results should be interpreted with caution. The patients/caregivers who read the PCIB seemed to benefit from it and found it useful and were consequently well informed about the ADRs associated with aripiprazole treatment and their spontaneous reporting requirements. Of the various highlighted ADRs, patients/caregivers seemed to be more vigilant about weight gain and started monitoring their weight regularly while receiving aripiprazole treatment. This is clinically relevant, as antipsychotic-related weight gain and associated metabolic AEs often increase the risk of cardiovascular disease, which might negatively impact treatment adherence and, in turn, efficacy [15-17].

It is difficult to comment on the reach of the survey as it is possible that the RM tools did reach the HCPs and/or the patients/caregivers, but they voluntarily chose not to respond to the survey invitation. The low response to the survey affects the precision of the survey results, possibly resulting in study bias, and making it difficult to generalize the study results.
Overall, the results indicate that, despite efforts to distribute the RM tools widely, the number of respondents was quite low, and the tools appear to have had limited utility and effectiveness in terms of HCP and patient/caregiver awareness and education. Therefore, the MAH proposed to the EMA-PRAC that the aRMMs be discontinued. The EMA-PRAC reviewed the findings from this study and the existing routine safety measures (aggregate reporting, signal management activities, and distribution of up-to-date summary of product characteristics and patient information leaflet) and issued an opinion indicating that the aRMMs could be discontinued (EMA application number II/0122). Thus, the aripiprazole RM tools were discontinued with effect from 10 November 2016, and the aripiprazole RMP was amended accordingly. Nonetheless, these findings are important and highlight the need to educate HCPs and patients/caregivers about the importance of similar survey-based studies.

One of the key limitations of this study was the extremely low response rate from HCPs and even lower response rate from patients/caregivers during the first round of HCP recruitment. During the study, it was believed that this could be due to the randomized distribution lists, intended to ensure an unbiased selection of participants. Consequently, the randomization step was removed for the second round, and all HCPs on the distribution list were invited, via three rounds of postal invitations, to participate in the survey. This was deemed appropriate to increase the response rate. However, the $\mathrm{HCP}$ response rate improved only marginally, as not all participants completed the survey.

Multiple factors might have contributed to the low response to the survey. One could be the use of postal 
invitations rather than electronic media. Initially, invitations to participate in the survey were sent via post. This was the preferred method agreed with local regulatory authorities for the distribution of the RM tools, possibly because of the limited alternatives of electronic communication at the time of rolling out the RM tools in 2013 and 2014. Despite the best efforts of the MAH in obtaining the email addresses of the HCPs, they were limited by strict privacy laws within the EU [18]. Thus, it was not possible to reach all HCPs on the distribution list that was initially approved by the competent authorities for the implementation of aRMMs. Therefore, the MAH and/or the clinical research organization had to rely on postal invitations, thereby limiting the receipt and acknowledgment from the intended recipient. This seems plausible as there was a good response in Spain, where email addresses were available for most of the HCPs, demonstrating a more pragmatic two-way communication approach.

Other possible reasons for the low response from HCPs could be deduced from the postal invitations returned with the following comments: the HCP was no longer available at the hospital, the HCP contact details were incorrect, or the HCP had no patients with BPI mania at the time of the survey. For example, one of the invited HCPs from Ireland stated, "I only have 1 or 2 patients, and usually none at all, diagnosed with BPI mania at any time and, obviously, not all patients are usually prescribed aripiprazole." It is also possible that HCPs and patients/caregivers voluntarily chose not to respond to the survey, despite receiving the tools, though we could not document this. This disinterest could be because of the perception that survey-based studies have limited scientific impact compared with clinical trials [11]. The HCPs from Slovenia and Denmark were unwilling to participate and did not respond to the survey invitation. All this clearly demonstrates the difficulties in conducting such surveys, despite the best operational efforts.

The final recipients of the RM tools were determined by the competent authorities in the participating countries. However, their approach to approving the distribution lists varied, which could have led to a low response rate. For example, in some countries, the HCP distribution lists included only child or adolescent psychiatrists, whereas others included general practitioners who may (but not necessarily) have the knowledge to treat patients with BPI mania. This meant the distribution lists across the region varied considerably, which in turn made it difficult to recruit the intended HCP population who may have received the tools and treated the appropriate patient population for this indication with aripiprazole.

Moreover, the survey study was classified as a PASS, which mandates the MAH to follow the stringent EU regulations as well as country-specific local regulations.
Additionally, the MAH had to adapt the RM tools with respect to content and assessment as per local health practices of the participating countries and gain approval of those. The timelines for these activities varied widely, ranging from 30 days to $\geq 6$ months. This eventually appeared to delay the tool distribution and hampered the real-time assessments of the tools' effectiveness, thereby jeopardizing the study. Another plausible reason could be that the compensation provided for PASS participation was comparatively lower than that provided in clinical trials (due to the lack of additional medical procedures), which could have resulted in a degree of disinterest among HCPs [11]. Further, because such survey studies have a PASS designation, HCPs in countries such as Spain require ethics submission and approval from their institutions, along with an institutional contract to participate in such studies. In addition, countries such as Germany have stricter disclosure requirements, including a written agreement with the HCP being compensated, HCP contact details, and registration number, which are shared with the payer organizations. Even if we ignore the low compensation, HCPs may find it tedious to complete the additional disclosure procedures and negotiate contracts with their institutions, which may have led to low participation.

Another challenge in this study was the low prevalence of BPI mania in the targeted age range [3]. Though the sample size was calculated, it was challenged by precision in estimating the size of the HCP population treating adolescents with this indication. The lack of standard diagnostic criteria globally [19] might have further complicated the diagnosis, given the symptoms of BPI mania overlap with other psychiatric disorders. Literature shows considerable variability in BPI diagnosis for the same set of manifested symptoms across regions, e.g. in the USA and the UK [19]. In addition, there exists a conservative approach towards the use of antipsychotics in the younger population in different parts of the world [20]. The pharmacological interventions available for psychiatric disorders in children or adolescents are also very limited [15]. Hence, off-label use of antipsychotic drugs in this population is common [16].

The low response from HCPs translated into a low response rate from patients/caregivers. The MAHs were unable to recruit patients directly through prescription databases because of the strict privacy laws in the EU. As such, the MAH had to rely on the prescribing HCP to distribute the RM tools to aripiprazole-treated patients and subsequently recruit patients to participate in the survey. To recruit patients, it was important to obtain informed consent, which could have hampered recruitment. The patients and caregivers were only recruited from three countries (the UK, Spain, and Norway). This might be 
because HCPs from the other countries did not want to seek informed consent from patients/caregivers.

Not limited to this survey study, other reasons for low participation from HCPs and patients/caregivers could be the lack of available time/resources, complexity of forms and procedures for participation, the inability of HCPs to identify treated patients during the study period or nonavailability of the indicated patient population at the time of study, and mandatory requirements such as sign-off trainings or workshops for HCPs.

The limitations of this study also include lack of standard thresholds to measure the knowledge of treatment risks. Further, most participants who enroll in knowledge and behavior surveys respond to the questions by recalling specific events rather than all events, thus introducing recall bias [10].

For successful survey-based studies in the future, it is imperative to use innovative ideas to ensure a wider reach to participants and optimize response rates. Utilizing digital or web-enabled RM tools to capture real-time data, more user-friendly formats, automated reminders, and engaging patient education and advocacy organizations might make such surveys more effective.

The response rate might also be improved by conducting a pre-study feasibility assessment of the market size and recruitment options, seeking advice from key opinion leaders with core experience in treating the relevant patient population, patient consultation when creating educational materials, and possibly including some mandates such as sign-off trainings while providing and receiving the treatment.

An example of effective aRMMs for a drug product for treatment of an orphan disease condition was implemented by the same MAH. In this case, the MAH not only ensured that all HCPs and patients/caregivers had access to their respective educational materials but also made it mandatory for HCPs to complete and sign-off the training before prescribing the drug to patients for the first time [21]. It is important to note that the design and execution of aRMMs depends on the indication, severity, and nature of the identified risks associated with the treatment.

In addition, there is a need for a centralized body to provide ethics approval to conduct a survey-based aRMMs effectiveness study across different countries. This could substantially reduce the operational challenges such as variations in approval time and the content of aRMMs material among participating countries. Detailed regulatory guidance is also required on the evaluation of aRMM effectiveness to avoid inconsistency.

There is a substantial need to better understand the limitations of survey-based studies and identify best practices around their design and implementation. This would improve the evaluation of aRMM effectiveness in the future and may provide better insight into the impact of aRMMs in a real-world setting. Nonetheless, it should be noted that, even when best efforts are made, the success of survey-based studies would still depend heavily on the interest of participants. Unless there is a strong driving factor or mandate to complete surveys, success remains unpredictable, as potential participants often perceive survey completion as tedious [22]. An awareness campaign to sensitize patients, caregivers, and HCPs emphasizing the importance of these survey-based studies to motivate them to participate is highly warranted.

It is necessary to implement aRMMs if the risks associated with the treatment cannot be controlled via routine RM measures. However, successful implementation of aRMMs requires collaboration between stakeholders, the healthcare system, and patients, along with clearly defined objectives and measures of their effectiveness [22].

\section{Conclusion}

Overall, considering the results of this study, we believe there is a need to implement an innovative framework to conduct survey-based studies to reduce the numerous operational challenges and encourage potential participants (HCPs and patients/caregivers) to engage in such studies. Additional regulatory guidance on these studies will be beneficial to define the desired elements and outcomes of aRMMs.

Acknowledgements The effectiveness survey was conducted by Pope Woodhead and Associates Ltd., UK, on behalf of Otsuka Pharmaceuticals Europe Ltd., UK. The authors thank Dr. Beth Pulaski (Otsuka Pharmaceutical Development and Commercialization Inc., USA) for her scientific contribution towards this paper. Medical writing and editorial support was provided by Mayuri Shinde, Dr. Vaibhav Deshpande, and Dr. Payal Bhardwaj (Tata Consultancy Services Ltd., India) and was funded by Otsuka Pharmaceuticals Europe Ltd., UK.

\section{Compliance with Ethical Standards}

Funding This study (ENCePP registration number: EUPAS6083) was funded by Otsuka Pharmaceutical Europe Ltd.

Ethics Ethics committee approval was obtained in Slovenia (Komisija Republike Slovenije za Medicinsko Etiko), Spain (Comité Ético de Investigación Clínica del Area de Salud de Burgos y Soria), Germany (Ethik-Kommission bei der Landesärztekammer Hessen), and Italy (Comitato Etico Azienda Ospedaliero Universitaria di Cagliari) as per local requirements. For the remaining countries, approval from an ethics committee was not required to conduct the surveys.

Conflicts of interest Wally Landsberg is an employee of Otsuka Europe Development and Commercialization Ltd., Wexham, UK. Emiel van Heumen, Antonia Coppin-Renz, and Uli Geis are employees of Otsuka Europe Development and Commercialization 
Ltd., Frankfurt am Main, Germany. Timothy Peters-Strickland and Mirza Rahman are employees of Otsuka Pharmaceutical Development and Commercialization, Inc, Princeton, NJ, USA. Imad AlDakkak is an employee of Pope Woodhead and Associates Ltd., Cambridgeshire, UK.

Open Access This article is distributed under the terms of the Creative Commons Attribution-NonCommercial 4.0 International License (http://creativecommons.org/licenses/by-nc/4.0/), which permits any noncommercial use, distribution, and reproduction in any medium, provided you give appropriate credit to the original author(s) and the source, provide a link to the Creative Commons license, and indicate if changes were made.

\section{References}

1. Muneer A. The treatment of adult bipolar disorder with aripiprazole: a systematic review. Cureus. 2016;8:e562.

2. Dell'Aglio JC Jr, Basso LA, Argimon II, Arteche A. Systematic review of the prevalence of bipolar disorder and bipolar spectrum disorders in population-based studies. Trends Psychiatry Psychother. 2013;35:99-105.

3. Ryles F, Meyer TD, Adan-Manes J, MacMillan I, Scott J. A systematic review of the frequency and severity of manic symptoms reported in studies that compare phenomenology across children, adolescents and adults with bipolar disorders. Int J Bipolar Disord. 2017;5:4.

4. ABILIFY (aripiprazole) Summary of product characteristics. Tokyo, Japan; Otsuka Pharmaceutical Co.; Published May, 2017. http://www.ema.europa.eu/docs/en_GB/document_library/EPAR _-_Product_Information/human/000471/WC500020170.pdf. Acc essed 10 July 2017.

5. Biederman J, Mick E, Spencer T, Doyle R, Joshi G, Hammerness $\mathrm{P}$, et al. An open-label trial of aripiprazole monotherapy in children and adolescents with bipolar disorder. CNS Spectr. 2007;12:683-9.

6. Keck PE Jr, Marcus R, Tourkodimitris S, Ali M, Liebeskind A, Saha A, et al. A placebo-controlled, double-blind study of the efficacy and safety of aripiprazole in patients with acute bipolar mania. Am J Psychiatry. 2003;160:1651-8.

7. Heads of Medicines Agency (HMA) and European Medicines Agency (EMA) (2012e) Guideline on good pharmacovigilance practices (GVP). Module XVI - Risk minimisation measures: selection of tools and effectiveness indicators (Rev 2). EMA/ 204715/2012 Rev 2. London: EMA. 2017. http://www.ema. europa.eu/docs/en_GB/document_library/Scientific_guideline/ 2014/02/WC500162051.pdf. Accessed 12 July 2017.

8. Agyemang E, Bailey L, Talbot J. Additional risk minimisation measures for medicinal products in the European Union: A review of the implementation and effectiveness of measures in the United Kingdom by one marketing authorisation holder. Pharm Med. 2017;31:101-12.
9. European Commission. Directive 2010/84/EU of the European Parliament and of the Council of 15 December 2010 amending, as regards pharmacovigilance, Directive 2001/83/EC on the Community code relating to medicinal products for human use. 2010. http://eur-lex.europa.eu/LexUriServ/LexUriServ.do?uri=OJ:L: 2010:348:0074:0099:EN:PDF. Accessed 22 Jan 2018.

10. Banerjee A, Zomerdijk IM, Wooder S, Ingate S, Mayall SJ. Postapproval evaluation of effectiveness of risk minimisation: methods, challenges and interpretation. Drug Saf. 2014;37:33-42.

11. Madison T, Arias A, DiSantostefano R, Gilsenan A, Matus D, Primatesta $\mathrm{P}$, et al. Evaluating the effectiveness of additional risk minimisation measures via surveys in Europe: challenges and recommendations. Endorsed by ISPE Board of Directors November 14, 2016. https://pharmacoepi.org/pub/f46953df-de6931e7-8f74-725bd7fa685f. Accessed 10 July 2017.

12. Zomerdijk IM, Sayed-Tabatabaei FA, Trifirò G, Blackburn SC, Strukenboom MC, Stratus SM. Risk minimization activities of centrally authorized products in the EU: a descriptive study. Drug Saf. 2012;35:299-314.

13. Keddie S. A descriptive study of additional risk minimization measures included in risk management plans reviewed by the United Kingdom Regulatory Authority. Pharm Med. 2013;27:25-34.

14. Observational Study Protocol 31-13-300, ENCEPP/SDPP/6083. http://www.encepp.eu/encepp/openAttachment/fullProtocol/6099. Accessed 15 Jan 2018.

15. Maayan L, Correll CU. Weight gain and metabolic risks associated with antipsychotic medications in children and adolescents. J Child Adolesc Psychopharmacol. 2011;21:517-35.

16. Persico AM, Arango C, BuitelaarJK Correll CU, Glennon JC, Hoekstra PJ, et al. Unmet needs in paediatric psychopharmacology: present scenario and future perspectives. Eur Neuropsychopharmacol. 2015;25:1513-31.

17. Wu RR, Zhao JP, Jin H, Shao P, Fang MS, Guo XF, et al. Lifestyle intervention and metformin for treatment of antipsychotic-induced weight gain: a randomized controlled trial. JAMA. 2008;299:185-93.

18. Nkeng L, Cloutier AM, Craig C, Lelorier J, Moride Y. Impact of regulatory guidances and drug regulation on risk minimization interventions in drug safety: a systematic review. Drug Saf. 2012;35:535-46.

19. Renk K, White R, Lauer BA, McSwiggan M, Puff J, Lowell A. Bipolar disorder in children. Psychiatry J. 2014;2014:928685.

20. Huskamp HA, Horvitz-Lennon M, Berndt ER, Normand ST, Donohue JM. Patterns of physician antipsychotic prescribing to young children. Psychiatr Serv. 2016;67(12):1307-14.

21. JINARC (tolvaptan) Summary of product characteristics. Otsuka Pharmaceutical Co.; Published May, 2017. http://www.ema. europa.eu/docs/en_GB/document_library/EPAR_-Product_Infor mation/human/002788/WC500187921.pdf. Accessed 10 July 2017.

22. Collins J, Bonneh-Barkay D. Considerations for successful riskminimisation strategies in the EU. Pharm Med. 2016;30:257-61. 